MATEC Web of Conferences 22, 03008 (2015)

DOI: $10.1051 /$ matec conf/ 20152203008

(C) Owned by the authors, published by EDP Sciences, 2015

\title{
A New Optimal Control Algorithm for Quad-rotor Helicopter with State Constraints via Sliding-mode Control
}

\author{
Jing Zhao, Pu Yang*, Zecheng Zhang \& Jianwei Liu \\ College of Automation Engineering, Nanjing University of Aeronautics and Astronautics, Nanjing, Jiangsu, \\ China
}

\begin{abstract}
This paper proposes a strategy of a new optimal sliding-mode control for flight control system with state constraints so that the system guarantees the optimal performance index. Besides, the strategy ensures strong robustness to the internal parametric uncertainty and the external disturbances. In order to have fast transient response speed as well as good tracking accuracy, the integral of the time multiplied by the absolute displacement tracking error is introduced as the performance index. By analyzing the state constraints which are specifically the velocity tracking error constraint and the acceleration tracking error constraint, and the performance index, the parameters of sliding-mode surface and control law are obtained. Finally, the authors conduct the semi physical simulation on Qball-X4 quad-rotor helicopter, showing the effectiveness of the proposed strategy.
\end{abstract}

Keywords: flight control system; state constraint; sliding-mode control; optimal control; quad-rotor helicopter

\section{GENERAL INSTRUCTIONS}

UAV (unmanned aerial vehicle) has an importan value of application both in military and civil fields for its characteristics such as low risk, good flexibility, high reliability and strong adaptability. However, more and more requirements are increased with the flight control system. A lot of control strategies are put forward to meet multiple performance objectives, such as closed-loop stability, fast transient response and precise tracking. Among them, the sliding-mode control is widely used in flight control system [1-4].

Due to the changes of the flight environment during flight and the inherent uncertainty in flight control system which may cause the decline of flight control system, even the instability of the aircraft, thus, the robustness and efficiency are required. The sliding-mode control provides a robust and effective method of controlling due to its outstanding advantage that the system dynamics will be insensitive to parameter variations and disturbances once the system state reaches a sliding surface [5-7].

Besides, the actual flight control system is always subject to various physical constraints. In [8], an active fault accommodate strategy is put forward for flight control system in the presence of input constraints. This method improves the tracking performance and eliminates the influences of actuator faults. In order to deal with the input constraints and severe actuator failures, [9] proposes a novel control scheme in which the control allocation method is incorporated with model reference adaptive control, realizing the global stability of the system and the asymptotic state tracking. Apart from input constraints, the flight control system also suffers from state constraints due to the *Corresponding author: ppyang@,nuaa.edu.cn limited input torque, working range and measurement speed. However, there are rare researches on state constraint in flight control system. A two-loop performance-oriented control approach is given for a class of multiple-input-multiple-output (MIMO) system with input saturation and state constraints in [10], getting fast transient response speed as well as good steady-state tracking accuracy without violating constraints. In [11], a global optimal sliding-mode control algorithm is proposed for non-linear system to conquer the acceleration constraint. This algorithm optimizes the performance index. Although these papers mentioned earlier research the state constraint to some degree, they are not combined with the flight control system. As the flight control system is subject to parametric uncertainty and external disturbances, it is hard to make sure that the tracking error converges monotonically as soon as possible without violating state constraints when the initial states are far away from the desired trajectory.

Therefore, based on the actual flight control system, this paper presents a strategy of optimal sliding-mode control for non-linear flight control system which is subject to parametric uncertainty, external disturbances, and state constraints which are respectively the state tracking error constraints. The integral of time multiplied by the absolute displacement tracking error is introduced as the performance criterion. By determining the parameters of sliding-mode surface, the optimal control strategy can be obtained. The proposed strategy can ensure that the displacement tracking error converges to zero without overshoots and minimizes the performance criterion to achieve good tracking accuracy and fast transient response speed based on the condition of state constraints. 


\section{MATEC Web of Conferences}

This paper is organized as follows. Problem formulation is presented in Section 2. Besides, the state constraints, more specifically, the velocity tracking error constraint and the acceleration tracking error constraint are analyzed and formulated. Then the sliding-mode control strategies are discussed in Section 3. In this section, the optimal parameters of sliding-mode surface and control law are obtained. In Section 4, the semi physical simulation is conducted on Qball-X4 which is produced by Quanser Company. The effectiveness of the algorithm is proven by simulation. Finally, the conclusion is drawn in Section 5.

\section{PROBLEM FORMULATION}

The non-linear third-order flight control system is described as follows:

$$
x=F(x)+G(x) u+\Phi
$$

$$
F(x)=\left[x_{2} x_{3} f(x)+\Delta f\right]^{T}
$$

$G(x)=\left[\begin{array}{lll}0 & 0 & g(x)\end{array}\right]^{T}$

$$
\Phi=\left[\begin{array}{lll}
0 & 0 & d
\end{array}\right]^{T}
$$

Where $\left.{ }^{x=\left[x_{1}\right.} x_{2} x_{3}\right]^{T}$ is the state vector describing the state variables of the system which are respectively displacement, velocity and acceleration. $u$ is the actuator input. $g(x)$ and $f(x)$ are the non-linear functions of state. $\Delta f$ and $d$ especially represent the parametric uncertainty and the external disturbances satisfying $|\Delta f+d| \leq \varphi$.

The desired trajectory that system (1) should track is $x_{d}=\left[\begin{array}{lll}x_{1 d} & x_{2 d} & x_{3 d}\end{array}\right]^{T}$ where $x_{1 d}=x_{2 d}$ and $x_{2 d}=x_{3 d}$ Furthermore, $x_{3 d}$ is differentiable. The tracking errors of the system are represented by vector $e=\left[e_{1} e_{2} e_{3}\right]^{T}=x-x_{d}$, where $e_{1}$ represents the displacement tracking error. At the initial time, the tracking errors are defined as $e_{0}=\left[\begin{array}{lll}e_{10} & 0 & 0\end{array}\right]^{T}$.

We consider the flight control system which suffers from two types of state constraints:

(1)The velocity tracking error constraint is shown as follows:

$$
\left|e_{2}(t)\right| \leq v_{\max }
$$

(2)The acceleration tracking error constraint is shown as follows:

$\left|e_{3}(t)\right| \leq a_{\max }$

\section{SLIDING-MODE CONTROL STRATEGY}

In this section, the parameters of sliding-mode surface are selected, and the control law is designed.

The sliding surface is chosen as follows:

$$
s= \begin{cases}\alpha+\beta t+e_{3}+A e_{2}+B e_{1} & t \leq t_{f} \\ e_{3}+A e_{2}+B e_{1} & t>t_{f}\end{cases}
$$

Where, $\alpha, \beta, A, B$ are the scalar constants to be determined, and $\alpha+\beta t$ is the global sliding-mode factor. $t_{f}$ is the switching time of sliding-mode surface when $\alpha$ and $\beta$ satisfy $\alpha+\beta t_{f}=0$.

At the initial time $t=0$, the representative point of the system belongs to the sliding-mode surface satisfying $s=0$. So it can be obtained as follows:

We obtain it as follows:

$$
\alpha+B e_{10}=0
$$

$$
\alpha=-B e_{10}
$$

From (7), at the time $t \in\left(0, t_{f}\right)$, we obtain it as follows:

$$
\begin{aligned}
& \alpha+\beta t+e_{3}+A e_{2}+B e_{1}=0 \\
& \ddot{e_{1}}+A \dot{e}_{1}+B e_{1}+\alpha+\beta t=0
\end{aligned}
$$

While during the time $t \in\left(t_{f}, \infty\right)$, we obtain it as follows:

$$
\ddot{e_{1}}+A \dot{e_{1}}+B e_{1}=0
$$

In order to make the displacement tracking error converge to zero monotonically without overshoot as soon as possible, the characteristic equation (12) should have a pair of equal real roots [12]. Therefore, we obtain it as follows:

$$
A=2 B^{\frac{1}{2}}
$$

Then solving (11) and (12) with (9) and (13), for the time $t \in\left(0, t_{f}\right)$, the tracking errors can be described as follows:

$e_{1}=-\left(2 \beta B^{-\frac{2}{3}}+\beta B^{-1} t\right) e^{-B^{\frac{1}{2}} t}+2 \beta B^{-\frac{2}{3}}+e_{10}-\beta B^{-1} t$

$e_{2}=\dot{e}_{1}=\beta\left(1+B^{\frac{1}{2}} t\right) B^{-1} e^{-B^{\frac{1}{2} t}}-\beta B^{-1}$

$e_{3}=e_{2}=-\beta e^{-B^{\frac{1}{2} t} t} t$

According to (14), (15) and (16), the tracking errors at the switching time $t=t_{f}$ can be obtained. Then we acquire the tracking errors for the time $t \in\left(t_{f}, \infty\right)$ as follows:

$e_{1}=\left[\beta\left(e^{B^{\frac{1}{2_{f}}}}-1\right)\left(t+2 B^{-\frac{1}{2}}\right) B^{-1}-e_{10} e^{B^{\frac{1}{2} t_{f}}}\right] e^{-B^{\frac{1}{2}} t}$ 


$$
\begin{aligned}
& e_{2}=\left[\beta B^{-\frac{1}{2}}\left(1-e^{B^{\frac{1}{2} t f}}\right)\left(1+B^{-\frac{1}{2}}\right)+e_{10} e^{B^{\frac{1}{2} t f}} B^{\frac{1}{2}}\right] e^{-B^{\frac{1}{2}} t} \\
& e_{3}=\left[-e_{10} B e^{B^{\frac{1}{2}} t f}+\beta\left(e^{B^{\frac{1}{2}} t f}-1\right) t\right] e^{-B^{\frac{1}{2}} t}
\end{aligned}
$$

We define $c$ as follows:

$$
c=B^{\frac{1}{2}} t_{f}
$$

Then $B$ can be described with $c$ as follows:

$$
B=\left(c \beta e_{10}^{-1}\right)^{\frac{2}{3}}
$$

In order to suppress the chatter of sliding-mode, the exponential reaching law is chosen as the control method, and the sign function is replaced by $R(s)=s /(|s|+\phi)$. So the selected sliding-mode surface is expected as follows:

$\dot{s}=-\lambda_{1} R(s)-\lambda_{2} s$

Solving (1) with (7) and (22), we can obtain the actuator input as follows:

$u=\frac{-\lambda_{1} R(s)-\lambda_{2} s}{g(x)}+\frac{x_{3 d}-f(x)-\Delta f-d-A e_{3}-B e_{2}-\eta \beta}{g(x)}$

Where $\eta= \begin{cases}\eta=1 & t \leq t_{f} \\ \eta=0 & t>t_{f}\end{cases}$

Considering the flight safety and flying qualities, the rapid response and control precision of flight control system should be guaranteed firstly. So the performance index is chosen as follows to minimize the integral of the time multiplied by the absolute displacement tracking error:

$$
J=\int_{t_{0}}^{\infty} t\left|e_{1}(t)\right| d t
$$

\subsection{Velocity tracking error constraint}

Theorem 1:The optimal value of (24) which is subject to (5) is achieved when $c_{o p 1} \rightarrow \infty$.

Proof: Solving (24) with (14) and (17), a function of $J$ is obtained as follows:

$$
J=\left|e_{10}\right|^{\frac{5}{3}}|\beta|^{-\frac{2}{3}}\left(c^{\frac{1}{3}}+\frac{c^{\frac{4}{3}}}{6}+3 c^{-\frac{2}{3}}\right)
$$

The derivative of $e_{2}(t)$ with respect to time is shown as follows: $e_{2}(t)=e_{3}(t)= \begin{cases}-\beta e^{-B^{\frac{1}{2}} t} t & t \leq t_{f}(26) \\ e^{-B^{\frac{1}{2}} t}\left[-B e_{10} e^{c}+\beta\left(e^{c}-1\right) t\right] & t>t_{f}\end{cases}$

The extreme value of $e_{2}(t)$ can be achieved when the time satisfies ${ }^{e_{2}\left(t_{v \max }\right)=0}$ :

$$
t_{v \max }=B e_{10} e^{c} \beta^{-1}\left(e^{c}-1\right)^{-1}
$$

Corresponding, the extreme value of $e_{2}(t)$ is shown as follows:

$$
e_{2}\left(t_{v \max }\right)=\beta B^{-1} e^{-c e^{c}\left(e^{c}-1\right)^{-1}}\left(1-e^{c}\right)
$$

Combining (28) and (21), we can obtain as follows:

$e_{2}\left(t_{v \max }\right)=e_{10}^{\frac{2}{3}}|\beta|^{\frac{1}{3}} c^{-\frac{2}{3}} e^{-c e^{c}\left(e^{c}-1\right)^{-1}}\left(1-e^{c}\right)$

Then (29) can be expressed as follows:

$e_{10}^{\frac{2}{3}}|\beta|^{\frac{1}{3}} c^{-\frac{2}{3}} e^{-c e^{c}\left(e^{c}-1\right)^{-1}}\left(1-e^{c}\right) \leq v_{\max }$

From (30), the extreme acceptable value of $|\beta|$ can be expressed as follows:

$|\beta| \leq v_{\max }{ }^{3} e_{10}^{-2} c^{2} e^{3 c e^{c}\left(e^{e}-1\right)^{-1}}\left(1-e^{c}\right)^{-3}$

Substituting (31) into (25), we acquire it as follows:

$J \geq v_{\max }{ }^{-2}\left|e_{10}\right|^{3} e^{-2 c e^{c}\left(e^{e}-1\right)^{-1}}\left(1-e^{c}\right)^{2}\left(c^{-1}+\frac{1}{6}+3 c^{-2}\right)(32)$

For any c, $\frac{d J}{d c} \leq 0$. Thus, the minimum of performance index can be achieved when it is shown as follows:

$c_{o p 1} \rightarrow \infty$

According to (9), (13), (20) and (31), the optimal value of other parameters in sliding-mode surface is shown as follows:

$$
\beta_{o p 1} \rightarrow \infty
$$

$A_{o p 1} \rightarrow \infty$

$B_{o p 1} \rightarrow \infty$

$\alpha_{o p 1} \rightarrow \infty$

$t_{f o p 1}=\frac{\left|e_{10}\right|}{v_{\max }}$ 


\section{MATEC Web of Conferences}

However, these parameters would result in the infinite control signal and the infinite acceleration tracking errors which are unacceptable in practical applications. Therefore, this method is not appropriate for the velocity tracking error only. Other factors should be taken into consideration to determine the feasible maximum value of $c_{o p 1}$

\subsection{Acceleration tracking error constraint}

Theorem 2:The optimal value of (24) which is subject to (6) is achieved when $c_{o p 1}=3 \sqrt{2}$.

Proof:At the switching time $t_{f}$, it is obviously shown as follows:

$\left|e_{3}\left(t_{f}\right)\right| \leq a_{\max }$

Thus:

$a_{\max } \geq e^{-c}\left(c \beta e_{10}^{-1}\right)^{\frac{2}{3}}\left|e_{10}\right|$

Therefore:

$|\beta| \leq\left[a_{\max } e^{-c} c^{\frac{2}{3}}\left|e_{10}\right|^{\frac{1}{3}}\right]^{\frac{3}{2}}$

From (16) and (19), function of $e_{3}(t)$ can be obtained:

$e_{3}(t)= \begin{cases}-\beta e^{-B^{\frac{1}{2}} t} t & t \leq t_{f} \\ e^{-B^{\frac{1}{2}} t}\left[-B e_{10} e^{c}+\beta\left(e^{c}-1\right) t\right] & t>t_{f}\end{cases}$

The derivative of function (42) with respect to time is shown as follows:

$e_{3}(t)= \begin{cases}-\beta e^{-B^{\frac{1}{2}} t}\left(1-B^{\frac{1}{2}} t\right) & t \leq t_{f} \\ e^{-B^{\frac{1}{2} t}}\left[-B^{\frac{3}{2}} e_{10} e^{k}+\beta\left(e^{k}-1\right)\left(1-B^{\frac{1}{2}} t\right)\right] & t>t_{f}\end{cases}$

During the time $t \leq t_{f}$, we can obtain the time $t_{\max }=B^{-\frac{1}{2}}$ when $e_{3}$ reaches the maximum according to (43). On the condition that $t_{\max 1}<t_{f}$, the maximum absolute value of $e_{3}(t)$ should be expressed as follows:

$e_{3}\left(t_{\max 1}\right)=-\beta e^{-1} B^{-\frac{1}{2}}$

If not, the maximum absolute value of $e_{3}(t)$ should be expressed as follows:

$e_{3}\left(t_{\max 1}\right)=e_{3}\left(t_{f}\right)$

During the time $t>t_{f}$, we can obtain the time when $e_{3}$ reaches the maximum as follows:

$$
t_{\max 2}=B^{-\frac{1}{2}}+B e_{10} e^{c}\left(e^{c}-1\right) \beta^{-1}
$$

Corresponding, the maximum absolute value of $e_{3}(t)$ should be expressed as follows:

$$
e_{3}\left(t_{\max 2}\right)=\left(e^{c}-1\right) e^{-c e^{c}\left(\mathrm{e}^{c}-1\right)^{-1}} \beta e^{-1} B^{-\frac{1}{2}}
$$

Apparently, $\left|e_{3}\left(t_{\max 1}\right)\right|>\left|e_{3}\left(t_{\max 2}\right)\right|$. So we obtain it as follows:

$|\beta| \leq \begin{cases}\left(a_{\max } e^{c} c^{-\frac{2}{3}} e_{10}^{-\frac{1}{3}}\right)^{\frac{3}{2}} & c<1 \\ \left(a_{\max } e c^{\frac{1}{3}} e_{10}^{-\frac{1}{3}}\right)^{\frac{3}{2}} & c \geq 1\end{cases}$

Solving (25) with (48), the function of performance index can be expressed with only one variable $c$ just as follows:

$J \geq \begin{cases}a_{\max }^{-1}\left|e_{10}\right|^{2} e^{-c}\left(\frac{1}{6} c^{2}+c+2\right) & c \leq 1 \\ a_{\max }{ }^{-1}\left|e_{10}\right|^{2} e^{-1}\left(\frac{1}{6} c+3 c^{-1}+1\right) & c>1\end{cases}$

The derivative of function(49)with respect to $c$ is shown as follows:

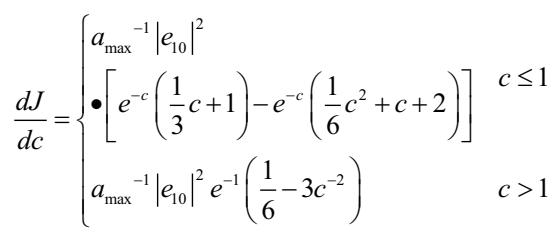

On the condition that $c \leq 1, \frac{d J}{d c}$ is always less than zero. When $c \leq 1$, the optimal value of $c$ is acquired as follows according to $\frac{d J}{d c}=0$ :

$c_{o p 2}=3 \sqrt{2}$

Corresponding, the optimal value of $\beta$ is shown as follows:

$\beta_{o p 2}=\left(a_{\max } e\right)^{\frac{3}{2}} e_{10}^{-\frac{1}{2}}(3 \sqrt{2})^{\frac{1}{2}}$

\section{SIMULATION}

In order to verify the effectiveness of the proposed optimal sliding-mode control strategy, we choose the Qball-X4 experimental platform which is produced by 
ICETA 2015

Quanser company as the research plant. As shown in Figure 1, the Qball-X4 experiment platform consists of helicopter body, Quarc acquisition card of aircraft data, target computer, power supply and real-time control software. Use the supporting software, the model built by Matlab/Simulink can be compiled to C code, and downloaded to real-time simulation system from PCI card through the parallel port [13]. Thus, the efficiency of the strategy can be verified by this simulation experiment.

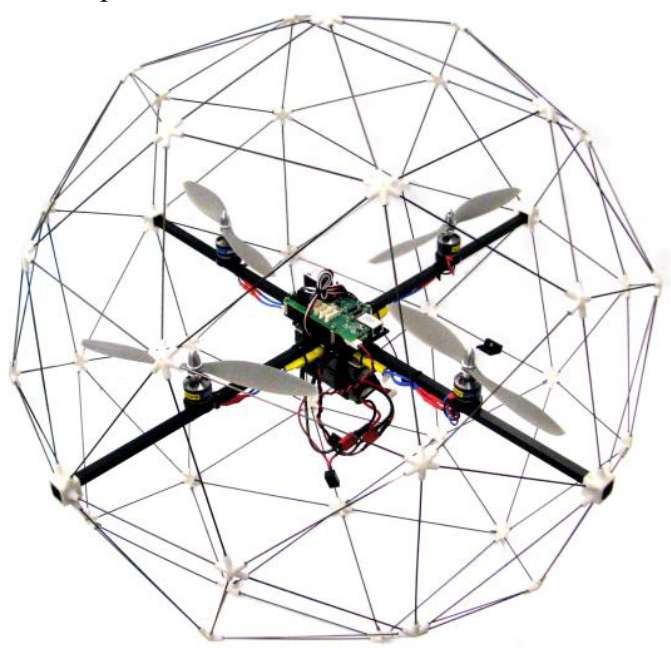

Figure 1. Quanser Qball-X4 experiment platform

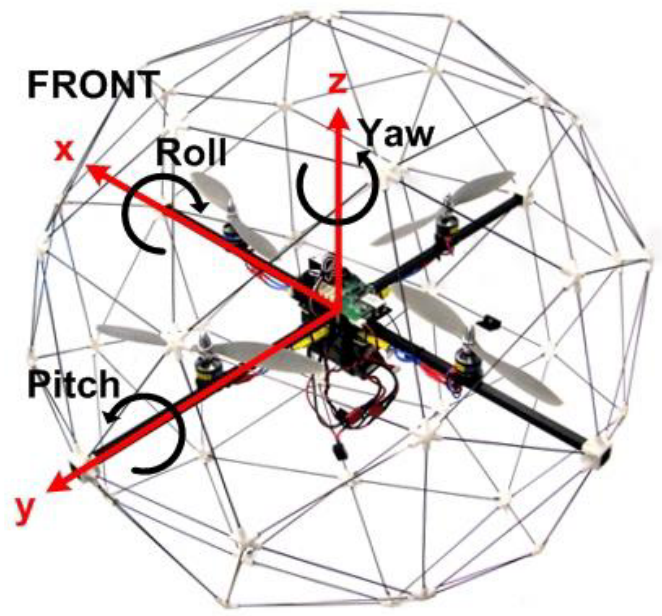

Figure 2. Qball-X4 axes and sign convention

As shown in Figure 2, the body coordinate system $O X Y Z$ is set up for convenience. Assume that the pitch angle $\theta$, roll angle $\phi$, yaw angle $\psi$ are all zero, Qball takes flat flight. Without loss of generality, only the control results of rotational movement in the $X$ axis are given. We select the displacement, velocity and acceleration in the $X$ axis as the system state variables which satisfy the following equations of state:

$x=F(x)+G(x) u+\Phi$

$\boldsymbol{F}(\boldsymbol{x})=\left[\begin{array}{lll}x_{2} & x_{3} & x_{1} x_{2}+\Delta f\end{array}\right]^{T}$

$\boldsymbol{G}(\boldsymbol{x})=\left[\begin{array}{lll}0 & 0 & 1\end{array}\right]^{T}$

$\boldsymbol{\Phi}=\left[\begin{array}{lll}0 & 0 & d\end{array}\right]^{T}$

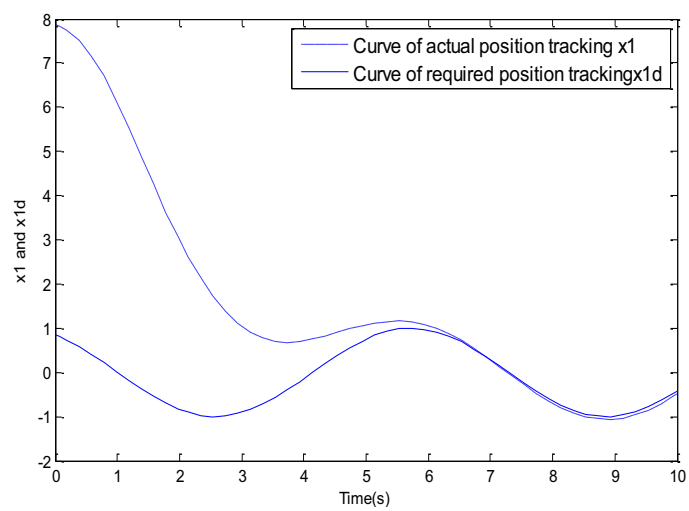

Figure 3. Curves of the actual and the required position tracking

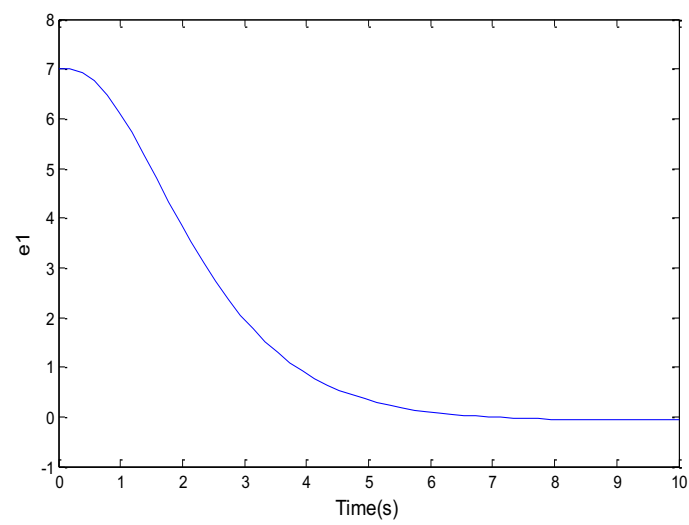

Figure 4. Curves of the replacement tracking error

Considering the common internal parametric uncertainty inside aircraft, and the external interference that aircraft may easily encounter during flight (such as the air flow disturbance), we assume that the parametric uncertainty is $\Delta f=0.39 \sin \left(x_{1} x_{2}+x_{3} t\right)$, and the flight control system suffers from the external disturbance that $d=0.6 \sin (10 \mathrm{t})$ at the same time.

The desired trajectory is $x_{1 d}=\sin (t+1.5)$, and at the initial time,the state vector of system is $x_{0}=\left[\begin{array}{lll}7.9975 & 0.07074 & -0.9975\end{array}\right]^{T}$.

On condition of the physical limits in flight control system, we assume that the state constraints are $e_{2} \leq 15 \mathrm{~m} / \mathrm{s}$ and $e_{3} \leq 3 \mathrm{~m} / \mathrm{s}^{2}$. We conduct the simulations which are subject to one state constraint each time. 


\section{MATEC Web of Conferences}

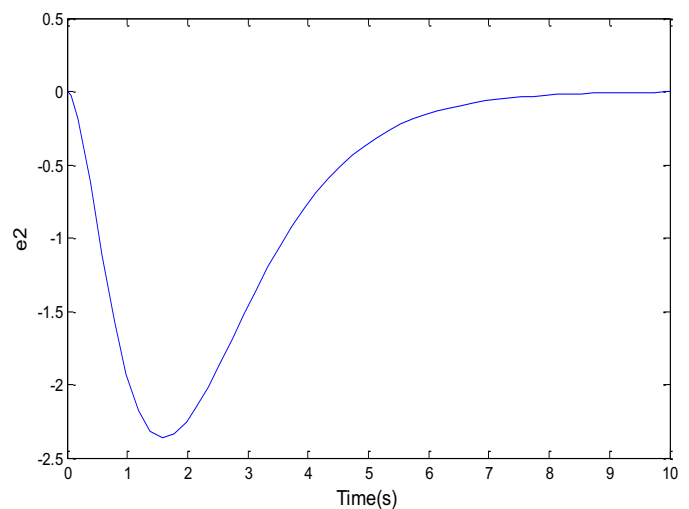

Figure 5. Curves of the velocity tracking error

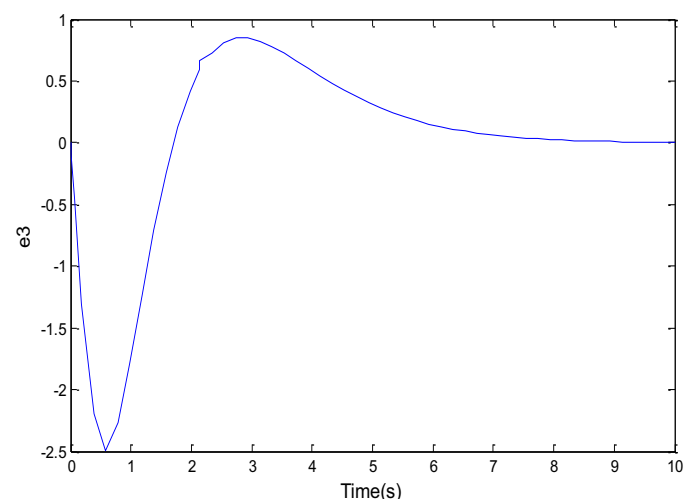

Figure 6. Curves of acceleration tracking error

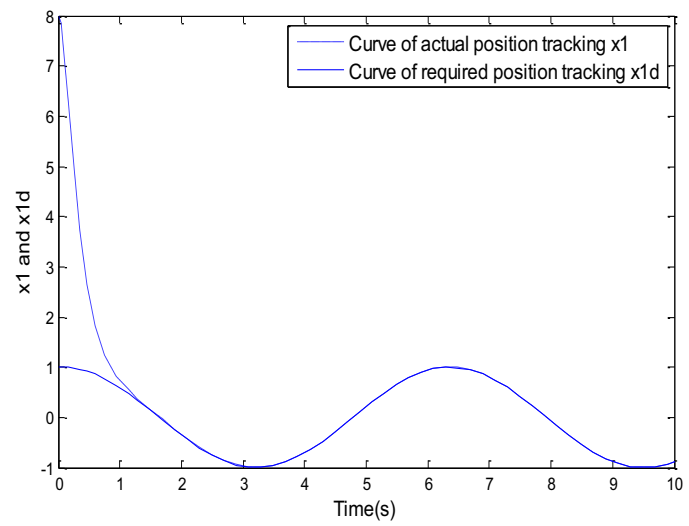

Figure 7. Curves of actual and required position tracking

Condition 1: The acceleration tracking error constraint is $e_{3} \leq 3 \mathrm{~m} / \mathrm{s}$

Figure 3 to Figure 6 show the simulation results on condition 1.

It is obvious that there is an initial position tracking error in the $X$ axis in Figure 3. However, Qball precisely tracks the target displacement in short time. The
Figure 4, Figure 5 and Figure 6 show that the choices of parameters of sliding-mode surface and control law ensure that the system's state tracking errors of replacement, velocity and acceleration converge to zero with accuracy rapidly. Especially, from Figure 6,the acceleration tracking error satisfies the state constraint $e_{3} \leq 3 \mathrm{~m} / \mathrm{s}^{2}$ all the time.

Condition 2: The velocity tracking error constraint is $e_{2} \leq 15 \mathrm{~m} / \mathrm{s}$.

Through several attempts, when $c_{o p}=20$, the corresponding sliding-mode surface and control law lead to the simulation results as follows that satisfy the velocity tracking error constraint. Figure 7 to Figure 10 are the simulation results on condition 2 .

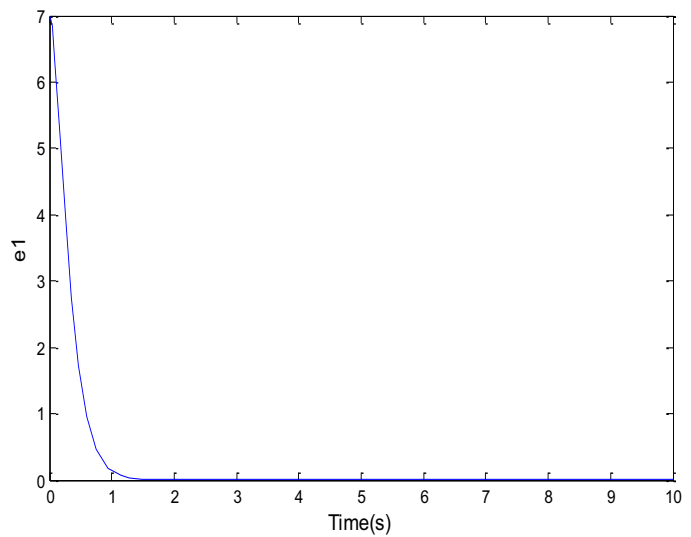

Figure 8. Curves of the replacement tracking error

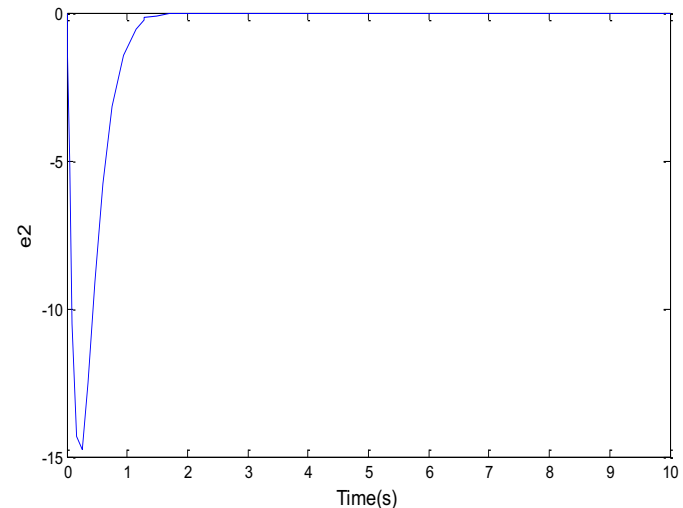

Figure 9. Curves of the velocity tracking error

From Figure 7, we can obtain that there is an initial position tracking error in the $X$ axis obviously, but the system is able to precisely track the target displacement in short time. The Figure 8, Figure 9 and Figure 10 indicate that the choices of parameters of sliding-mode surface and control law not only satisfy the velocity tracking error constraint $e_{2} \leq 15 \mathrm{~m} / \mathrm{s}$ but also make the system's state tracking errors of replacement, velocity and acceleration converge to zero rapidly. But clearly, there is a huge peak in curve of 
acceleration tracking error in Figure 10 which is unacceptable in reality. This situation would cause the flight control system out of control, or even a damage of vehicle. So when the system exists in the velocity tracking error constraint, the parameters of sliding-mode surface and control law should be selected with other factors, such as the acceleration tracking error constraint and the input constraint.

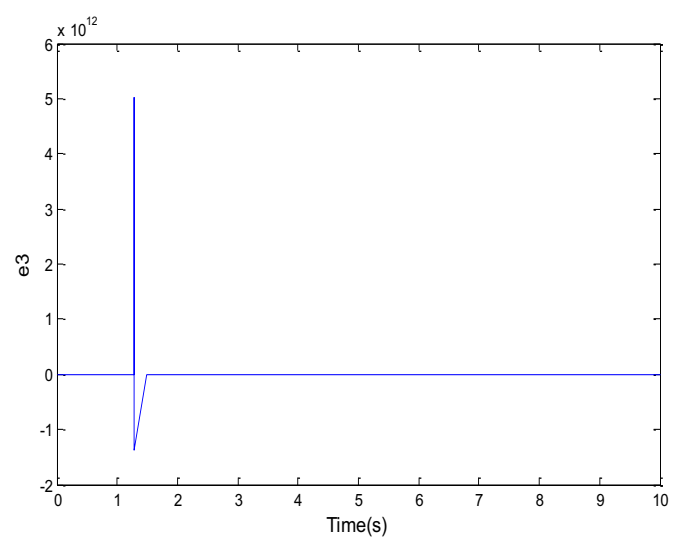

Figure 10. Curves of the acceleration tracking error

\section{CONCLUSIONS}

This paper presents a strategy of the optimal sliding-mode control for non-linear flight control system which is subject to parametric uncertainty, external disturbances, and state constraints which are specifically state tracking error constraints. The proposed strategy can ensure that the tracking error converges to zero and minimizes the performance criterion to achieve good tracking accuracy and fast transient response speed on the condition of state constraints.

However, this paper analyses the velocity tracking error constraint and acceleration tracking error constraint respectively, rather than together. Besides, other factors should be taken into consideration to determine the feasible parameters of sliding-mode surface and control law for velocity tracking error only. Therefore, further researches should be done subsequently.

\section{ACKNOWLEDGEMENT}

This paper is sponsored by National Natural Science Foundation of China (61203090), National Natural Science Foundation of China (61374130), Natural Science Foundation of Jiangsu Province (BK2012384) and Fund of National Engineering and Research Center for Commercial Aircraft Manufacturing (SAMC14-JS-15-053).

\section{REFERENCES}

[1] Mou, C. Changsheng, J. \& Rong, Mei. 2003. Design and simulation of optimal sliding mode fight control system. Electronics Optics \& Control. 10(3): 35-38.

[2] Mou, C. Rong, M. \& Bin, J. 2013. Sliding mode control for a class of uncertain MIMO nonlinear systems with application to near space vehicles. Mathematical Problems in Engineering.

[3] Jing, Z. Bin, J. \& Peng, S. 2013. Adaptive dynamic sliding mode control for Near Space Vehicles under actuator faults. Circuit Systems and Signal Processing. 32(5): 2281-2296.

[4] Erkan A. 2014. Flight control system design with high order sliding modes for nonlinear aircraft mode. 13th IEEE Workshop on Variable structure systems. Nantes, France.

[5] Laghrouche, S. Pleatan, F. \& Glumineau, A. 2007. Higher order sliding mode control based on integral sliding mode. Automatica. 43: 531-537.

[6] Utkin, V. 1977. Variable structure system with sliding-modes. IEEE Transactions on Automatic Control. 22(2): 212-222.

[7] Weibing, G. 1996. Theory and Design Method for Variable Structure Control. Beijing: Science Press.

[8] Yuying, G. Youming, Z. \& Bin, J. 2010. Multi-model-based flight control system reconfiguration control in the presence of input constraints. Proceedings of the 8th World Congress on Intelligent Control and Automation, Jinan, 5819-5824.

[9] Man, W. Jianying, Y. \& Yingxin Y et al. 2013. Fault-tolerant control for flight control systems in the presence of severe actuator failures and input constraints. Proceedings of the 32nd Chinese Control Conference, Xi'an, 6100-6105.

[10]Lu, L. \& Bin, Y. 2014. Online constrained optimization based adaptive robust control of a class of MIMO nonlinear systems with matched uncertainties and input/state constraints. Automatica. 50: 864-873.

[11]Feiyao, D. Humin, L. \& Haining, L et al. 2012. Optimal sliding-mode control for nonlinear systems subject to acceleration constraint. Control Theory \& Applications. 29(9):1223-1226.

[12]Chunsheng, L. \& Bin, J. 2009. Adaptive sliding-mode control for uncertain flight system with actuator dynamics. Journal of Applied Sciences-Electronics and Information Engineering. 27(4): 419-424.

[13]Quanser Consulting. 2005. Qball-X4 User Manual. Ontanio: Quanser Inc. 